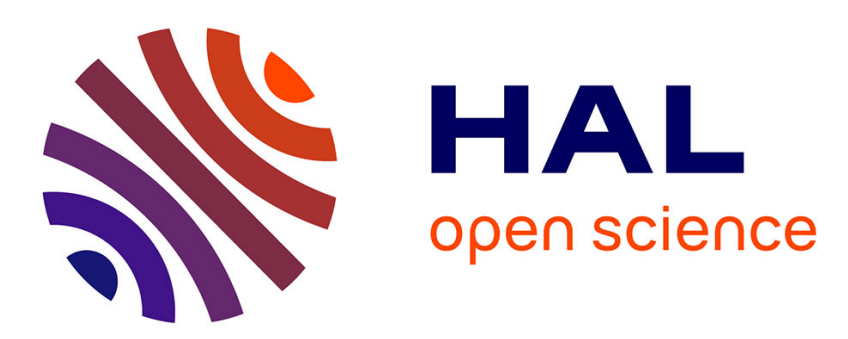

\title{
An example of a topologically non-rigid foliation of the complex projective plane
}

Loïc Teyssier

\section{To cite this version:}

Loïc Teyssier. An example of a topologically non-rigid foliation of the complex projective plane. International Mathematics Research Notices, 2011, 2011 (18), pp.4089-4104. 10.1093/imrn/rnq246 . hal-00423511v4

\section{HAL Id: hal-00423511 \\ https://hal.science/hal-00423511v4}

Submitted on 12 Nov 2012

HAL is a multi-disciplinary open access archive for the deposit and dissemination of scientific research documents, whether they are published or not. The documents may come from teaching and research institutions in France or abroad, or from public or private research centers.
L'archive ouverte pluridisciplinaire HAL, est destinée au dépôt et à la diffusion de documents scientifiques de niveau recherche, publiés ou non, émanant des établissements d'enseignement et de recherche français ou étrangers, des laboratoires publics ou privés. 


\title{
AN EXAMPLE OF A TOPOLOGICALLY NON-RIGID FOLIATION OF THE COMPLEX PROJECTIVE PLANE
}

\author{
LOÏC TEYSSIER
}

(C)2010, the author. Published by Oxford University Press. All rights reserved.

\begin{abstract}
We give here an explicit example of an algebraic family of foliations of $\mathbb{C} P^{2}$ which is topologically trivial but not analytically trivial. This example underlines the necessity of some assumptions in Y. Ilyashenko's rigidity theorem.
\end{abstract}

Institut de Recherche Mathématique Avancée

Université de Strasbourg (France)

\section{IntRoduction AND PRESENTATION OF THE RESUlT}

The aim of this article is to provide examples of algebraic foliations of $\mathbb{C} P^{2}$ which are not topologically rigid. A foliation on $\mathbb{C} P^{2}$ is defined in an affine chart $\mathbb{C}^{2}$ by a differential equation

$$
P(x, y) y^{\prime}=Q(x, y)
$$

where $P$ and $Q$ are complex polynomials. The space $\mathcal{C}_{d}$ of all such foliations with $P$ and $Q$ of a fixed degree $d$ is a complex projective space of finite dimension, endowed with the natural topology. A theorem of Yu. S. Ilyashenko [I] states that except maybe for a residual set, all foliations which are topologically conjugate are in fact analytically (thus homographically) conjugate, i.e. the generic foliation is topologically rigid. This result was later enhanced by various authors. In [S] A. Scherbakov showed that the set of topologically rigid foliations contains at least the complement of a real analytic set of $\mathcal{C}_{d}$. An improvement was given by X. GómezMont and L. Ortíz-Bobadilla [GO], then by L. Neto, P. Sad and P. Scárdua [NSS], showing that the set of all topologically rigid foliation is at least a Zariski-dense open set of $\mathcal{C}_{d}$. The argument boils down to proving that non-solvable holonomy representation is the typical behaviour, then applying Nakai's theorem $[\mathrm{N}]$ or using other results on density of orbits of (pseudo-)groups of local diffeomorphisms.

Examples of foliations of $\mathbb{C} P^{2}$ which are not topologically rigid do not abound. First examples of non-rigid foliations of $\mathbb{C} P^{2}$ can be deduced from the work of $\mathrm{N}$. Ladis [L], where the topological classification of generic homogeneous equations (1.1) is achieved.

We wish to present rather simple foliations which are not rigid. They belong to the class of Liouville-integrable foliations, whose holonomy representation is solvable. The main purpose of this paper is to prove their non-rigidity "by hand",

Date: November 2010. 
by building explicitely homeomorphisms between each members of the following family :

Theorem. Let $\Omega \subset \mathbb{C}$ be the domain defined by $\Omega:=\left\{\alpha \in \mathbb{C}:|\alpha|<\frac{1}{10} \sqrt{\frac{\pi}{2}}\right\}$. Each member of the family of linear differential equations in $\mathbb{C}^{2}$ :

$$
x^{3} y^{\prime}=y+x^{2}+\alpha x^{3} \quad, \alpha \in \Omega
$$

induces a foliation of $\mathbb{C} P^{2}$ which is not topologically rigid. More precisely, they all are topologically conjugate to each other whereas two equations with different $\alpha$ 's are not locally analytically conjugate near $(0,0)$.

In fact, a part of the second statement is given by P. M. Elizarov's result [E], where he describes the local topological classification of saddle-node equations, and by Martinet-Ramis' one [MR] about their local analytical classification. The cornerstone of the proof here is to build an homeomorphism of $\mathbb{C}^{2}$ which extends to the whole of $\mathbb{C} P^{2}$, which was not possible using Elizarov's purely local construction. For technical reasons and the sake of briefness it was necessary to choose $\Omega$ as given above, though the result should be valid for all $\alpha \in \mathbb{C} \backslash\{ \pm 1\}$. As a real map, the homeomorphisms introduced here are piece-wise affine but could be chosen $C^{\infty}$ outside $\{x=0\}$ by taking small perturbations. Yet according to a rigidity result of S. M. Voronin [V] the homeomorphism cannot be made $C^{1}$ in any neighbourhood of this line since otherwise the differential equations would be locally analytically conjugate.

Remark. This family of differential equations is not an unfolding in the sense of J.-F. Mattéi [M] since if there existed some germ of a holomorphic function $R$ such that $x^{3} \mathrm{~d} y-\left(y+x^{2}+\alpha x^{3}\right) \mathrm{d} x+R(x, y, \alpha) \mathrm{d} \alpha$ be integrable (as a 1-form) then one would obtain

$$
x^{3} \frac{\partial R}{\partial x}+\left(y+x^{2}+\alpha x^{3}\right) \frac{\partial R}{\partial y}=\left(1+3 x^{2}\right) R-x^{6} .
$$

On the one hand this equation admits a unique formal solution [T]. On the other hand so is the case for the differential equation $x^{3} f^{\prime}=\left(1+3 x^{2}\right) f-x^{6}$. Taking $R(x, y, \alpha):=f(x)$ thus yields the only possible solution. Unfortunately the latter power series is divergent.

\section{LOCAL STUDY OF THE SADDLE-NODE SINGULARITY}

Definition 2.1. When we say that two foliations are locally topologically conjugate (or simply topologically conjugate) near $(0,0)$ we mean that there exists an open neighbourhood $\Delta$ of $(0,0)$ and an orientation-preserving homeomorphism $\varphi: \Delta \rightarrow$ $\varphi(\Delta)$ fixing $(0,0)$ which sends a (trace on $\Delta$ of a) leaf of one foliation into a leaf of the other. If moreover $\varphi$ is an analytic map we say that the vector fields are locally analytically conjugate.

In the following we will study foliations $\mathcal{F}$ of $\mathbb{C} P^{2}$ on subdomains $\Delta$ of $\mathbb{C} P^{2}$ and we will implicitly mean that we consider the restriction of $\mathcal{F}$ to $\Delta$, i.e. the foliation whose leaves are the connected components of the trace on $\Delta$ of the leaves of $\mathcal{F}$. 
2.1. What is known. We first apply the linear change of variables $(y, \alpha) \mapsto$ $\left(-i \pi y, \sqrt{\frac{2}{\pi}} \alpha\right)$ in order to transform the family of equations (1.2) into

$$
x^{3} y^{\prime}=y-\frac{1}{i \pi} x^{2}-\frac{\alpha}{i \sqrt{2 \pi}} x^{3} .
$$

This change of variables is performed to simplify the upcoming computations. Let us denote by $\omega_{\alpha}$ the differential 1-form representing (2.1) which, in the affine chart $\mathbb{C}^{2}=\{(x, y)\}$, can be written as

$$
\omega_{\alpha}(x, y):=\left(y-\frac{1}{i \pi} x^{2}-\frac{\alpha}{i \sqrt{2 \pi}} x^{3}\right) \mathrm{d} x-x^{3} \mathrm{~d} y .
$$

Such a differential form is integrable and induces a foliation on $\mathbb{C} P^{2}$, which we denote by $\mathcal{F}_{\alpha}$, of saddle-node type at $(0,0)$, having exactly one separatrix passing through this point (namely $\{x=0\}$ ). Notice that all leaves of the foliation are transverse to the fibers of the natural projection

$$
\Pi: \quad(x, y) \mapsto x
$$

except for the separatrices $\{x=0\} \cup\{x=\infty\}$.

Let us recall two classical results which our argument is partly based upon.

Theorem 2.2. (Elizarov, $[\mathrm{E}])$ Let $E$ be the space of all saddle-node foliations given by differential forms $(y+R(x, y)) d x-x^{3} d y$, where $R$ is a germ of a holomorphic function at $(0,0)$ with $R(0,0)=0$ and $\frac{\partial R}{\partial y}(0,0)=0$. This space splits into $E_{1}$ and $E_{2}$ according to whether a given foliation has one or two separatrices through $(0,0)$.

(1) The quotient $E_{1} /$ top of local topological equivalence classes has cardinality 2.

(2) Two foliations in $E_{2}$ are locally topologically conjugate if, and only if, so are their "weak" holonomies, i.e. the holonomies computed on a transversal $\Pi^{-1}\left(x_{0}\right)$ by lifting through $\Pi$ a generator of the fundamental group of the second separatrix.

In fact the differential form (2.2) has such a simple form that it is integrable by quadrature, so its invariant of topological classification can be computed explicitly in terms of $\alpha$ (see the end of [MR] for a similar computation, or [T] for a more general one). It then turns out that when $\alpha^{2} \neq 1$ all foliations $\mathcal{F}_{\alpha}$, which belong to $E_{1}$, are mutually topologically conjugate. Besides $\mathcal{F}_{ \pm 1}$ belong to the other equivalence class.

Theorem 2.3. (Martinet-Ramis, [MR]) The quotient E/ana of local analytic equivalence classes is in one-to-one correspondence with the space $\mathbb{C} \times(\mathbb{C} \times c \mathbb{C}\{c\})^{2} / \sim$. The invariant of Martinet-Ramis is thus a 5-tuple $\mathcal{M}:=\left(\mu, \tau_{0}, \varphi_{0}, \tau_{1}, \varphi_{1}\right)$ where $\mu:=\frac{\partial^{2} R}{\partial x \partial y}(0,0)$ is the formal invariant, $\tau_{j}$ are scalars and $\varphi_{j}$ are germs of a vanishing holomorphic function at 0 , modulo the equivalence relation (with evident notations): $\mathcal{M} \sim \tilde{\mathcal{M}}$ if and only if $\mu=\tilde{\mu}, \tau_{j}=\lambda \tilde{\tau}_{j+k}, \varphi_{j}(c)=\tilde{\varphi}_{j+k}(\lambda c)$ for some $\lambda \in \mathbb{C}_{\neq 0}$ not depending on $j \in \mathbb{Z} / 2$ and for some $k \in \mathbb{Z} / 2$.

Remark 2.4. The space $E_{2}$ coincides with the space of foliations such that $\tau_{0}=$ $\tau_{1}=0$. 
The same computations as above yields that $\alpha$ is an analytic invariant, as we will see in the following section. More precisely one can choose $\mathcal{M}$ as follows :

$$
\begin{aligned}
\tau_{0} & :=1+\alpha \\
\tau_{1} & :=1-\alpha \\
\varphi_{j} & :=0 .
\end{aligned}
$$

Hence $\mathcal{F}_{\alpha}$ and $\mathcal{F}_{\beta}$ are always topologically conjugate (under the hypothesis $\alpha, \beta \notin$ $\{-1,1\})$ whereas they are analytically conjugate if, and only if, $\alpha=\beta$.

Remark 2.5.

(1) These invariants are not the "genuine" Martinet-Ramis invariants, which are more conventionally seen as gluing maps in the sectorial space of leaves, meaning the diffeomorphisms :

$$
\begin{aligned}
\psi_{j}^{\infty}: c & \mapsto c+\tau_{j} \\
\psi_{j}^{0}: c & \mapsto c \exp \left(i \pi \mu+\varphi_{j}(c)\right) .
\end{aligned}
$$

In the case where $\chi \in E_{2}$, i.e. $\psi_{j}^{\infty}=I d$, its weak holonomy is analytically conjugate to $\psi_{0}^{0} \circ \psi_{1}^{0}$, which is a map tangent to $e^{2 i \pi \mu} I d$.

(2) Elizarov's topological moduli space of $E_{1}$ is the set of all pairs $\left(\varepsilon_{0}, \varepsilon_{1}\right) \in$ $\{0,1\}^{2} \backslash\{(0,0)\}$ such that $\varepsilon_{j}=0$ if, and only if, $\tau_{j}=0$ where $(1,0)$ and $(0,1)$ are identified.

We propose here to build explicitly a local topological conjugacy between $\mathcal{F}_{\alpha}$ and $\mathcal{F}_{0}$ when $|\alpha|<\frac{1}{10}$. In fact one could achieve the same construction for any value of $\alpha$ but for the sake of concision we only retain this case. Before doing so we begin with describing the setting for any value of $\alpha$.

2.2. The sectorial decomposition and induced homemorphisms in the spaces of leaves. We split $\mathbb{C}^{2}$ into three parts :

$$
\mathbb{C}^{2}=\mathcal{V}^{+} \cup \mathcal{V}^{-} \cup\{x=0\}
$$

where the sectors $\mathcal{V}^{ \pm}$are, as usual, defined by

$$
\mathcal{V}^{ \pm}:=\left\{(x, y):\left|\arg x \mp \frac{\pi}{2}\right|<\frac{3 \pi}{4}\right\} .
$$

We denote $\mathcal{F}_{\alpha}$ the foliation induced on $\mathbb{C} P^{2}$ by $\omega_{\alpha}$ and define $\mathcal{F}_{\alpha}^{ \pm}$as the restriction of $\mathcal{F}_{\alpha}$ to $\mathcal{V}^{ \pm}$. We let $y_{\alpha, c}^{ \pm}$be the general solution of the differential equation $\omega_{\alpha}=0$ for $c \in \mathbb{C}$ :

$$
\left(y_{\alpha, 2}^{ \pm}\right): x \in \Pi\left(\mathcal{V}^{ \pm}\right) \mapsto \exp \left(-\frac{1}{2 x^{2}}\right)\left(c-\int_{ \pm 0 i}^{x}\left(\frac{1}{i \pi}+\frac{\alpha}{i \sqrt{2 \pi}} z\right) \exp \left(\frac{1}{2 z^{2}}\right) \frac{\mathrm{d} z}{z}\right)
$$

which are holomorphic functions. The integration here is done over a path linking $x$ to 0 in $\Pi\left(\mathcal{V}^{ \pm}\right)$and tangent to the half-line $\pm i \mathbb{R}_{\geq 0}$ at 0 . Notice that the intersection $\mathcal{V}^{+} \cap \mathcal{V}^{-}$is included in the node-part $\left\{\operatorname{Re}\left(x^{-2}\right)>\varepsilon>0\right\}$ of the saddle-node singularity of $\mathcal{F}_{\alpha}$, meaning that any leaf of $\mathcal{F}_{\alpha}^{ \pm}$, or of its restriction to any polydisc $\Delta$ centered at $(0,0)$, accumulates on $(0,0)$ over these sectors. On the contrary only one leaf accumulates on $(0,0)$ in the saddle-part $\left\{R e\left(x^{-2}\right)<-\varepsilon<0\right\}$. It is the leaf corresponding to $y_{\alpha, 0}^{ \pm}$and we will call it the sectorial weak separatrix. MartinetRamis invariants measure how going from one sector $\mathcal{V}^{ \pm}$to the other changes the value of $c$ while remaining on the same global leaf of $\mathcal{F}_{\alpha}$. In the special case we are 
considering they simply consist in the Stokes coefficients of the linear differential equation $\omega_{\alpha}=0$. One can easily check that

$$
\begin{aligned}
& (\forall \operatorname{Re}(x)<0) y_{\alpha, c}^{+}(x)=y_{\alpha, c+1+\alpha}^{-}(x) \\
& (\forall \operatorname{Re}(x)>0) y_{\alpha, c}^{-}(x)=y_{\alpha, c+1-\alpha}^{+}(x)
\end{aligned}
$$

so that

$$
\begin{aligned}
\tau_{0} & =1+\alpha \\
\tau_{1} & =1-\alpha .
\end{aligned}
$$

Indeed the value of the difference $y_{\alpha, c}^{+}-y_{\alpha, c}^{-}$can be obtained through Hankel's integral representation of $\frac{1}{\Gamma}$ :

$$
\int_{\gamma_{j}} z^{a} \exp \left(\frac{1}{2 z^{2}}\right) \frac{\mathrm{d} z}{z^{3}}=-\frac{2 i \pi}{\Gamma(a / 2)}\left(\frac{1}{2}\right)^{a / 2}(-1)^{a j}
$$

where $\gamma_{j}$ is a circle tangent at 0 to $i \mathbb{R}$ centered at $(-1)^{j}$.

We can assume without loss of generality that, up to changing slightly the aperture of the source and target sectors, $\varphi\left(\mathcal{V}^{ \pm} \cap \Delta\right) \subset \mathcal{V}^{ \pm}$. Hence, following the same argument as before, any homeomorphism $\varphi$ conjugating $\mathcal{F}_{\alpha}$ and $\mathcal{F}_{0}$ on some polydisc $\Delta$ induces (unique) homeomorphisms $\psi^{ \pm}$from the sectorial spaces of leaves of $\mathcal{F}_{\alpha}^{ \pm}$to the sectorial space of leaves of $\mathcal{F}_{0}^{ \pm}$. Since $\varphi$ must send sectorial weak separatrices of $\mathcal{F}_{\alpha}$ onto those of $\mathcal{F}_{0}$ the homeomorphisms $\psi^{ \pm}$shall fix 0 and we derive

$$
\begin{aligned}
\psi^{ \pm}: \mathbb{C} & \rightarrow \mathbb{C} \\
c & \mapsto \psi^{ \pm}(c) \\
0 & \mapsto 0
\end{aligned}
$$

such that

$$
\varphi\left(\left\{y=y_{\alpha, c}^{ \pm}(x)\right\}\right) \subset\left\{y=y_{0, \psi^{ \pm}(c)}^{ \pm}(x)\right\}
$$

and $\psi^{ \pm}$conjugate the actions of $c \mapsto c+\tau_{j}$. See figure 2.1. They will be called transverse homeomorphisms in the sequel as they completely determine the change in the transverse structure of the foliations.

On the converse our aim in the rest of Section 2 is to build special transverse homeomorphims conjugating the Stokes translations in order that they be realized in the $(x, y)$-space by a local homeomorphism $\varphi$. We will later extend it to the whole $\mathbb{C} P^{2}$ in Section 3.

2.3. The construction on $\mathbb{C}^{2}$. The strategy to build such a $\varphi$ consists in the following three steps.

(1) Finding two transverse homeomorphisms $\psi^{ \pm}$such that

$$
\begin{cases}\psi^{+}(c+1-\alpha) & =\psi^{-}(c)+1 \\ \psi^{-}(c+1+\alpha) & =\psi^{+}(c)+1 \\ \psi^{+}(0)=\psi^{-}(0) & =0 \\ \lim _{c \rightarrow 0, \infty} \frac{\psi^{ \pm}(c)}{c} & =1\end{cases}
$$




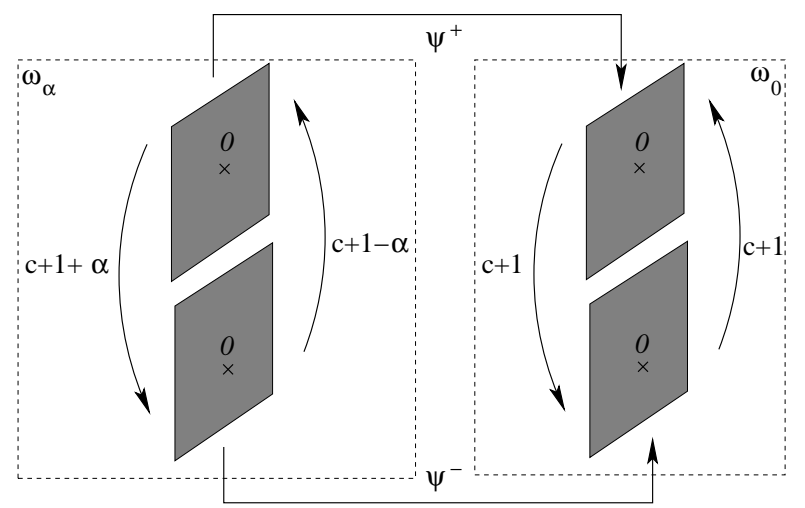

Figure 2.1. The induced homeomorphisms $\psi^{+}$and $\psi^{-}$between spaces of leaves

Notice the three first conditions are necessarily satisfied by any pair of induced homeomorphisms. Besides the first two relations imply that $\psi^{ \pm}=$ $I d+\eta^{ \pm}$where $\eta^{ \pm}$is 2-periodic.

(2) Finding a lift $\varphi^{ \pm}$of $\psi^{ \pm}$in the ambient space $\mathcal{V}^{ \pm} \cap \overline{\mathbb{D}} \times \mathbb{C}$ such that $\varphi^{+}=\varphi^{-}$ in $\mathcal{V}^{+} \cap \mathcal{V}^{-}$(thus defining a homeomorphism $\varphi$ on $\overline{\mathbb{D}}_{\neq 0} \times \mathbb{C}$ ).

(3) Ensuring that $\varphi$ extends continuously to $\{x=0\}$. For this we need the last condition in the above system.

To underline that fulfilling these three conditions is tricky we first prove the

Proposition 2.6. Assume that $\varphi$ is a local topological conjugacy between $\mathcal{F}_{\alpha}$ and $\mathcal{F}_{0}$ such that $\varphi$ preserves globally the fibers of $\Pi$. Then $\alpha=0$.

The meaning of this statement is that, unlike the analytical setting (see [MR]), topological conjugacies between non-analytically conjugate saddle-node foliations cannot be chosen of the form $(x, y) \mapsto(x, Y(x, y))$, nor even of the form $(x, y) \mapsto$ $(X(x), Y(x, y))$, which seriously complicates matters as we will see.

Proof. Let $\Delta$ be a polydisc on which $\varphi$ is realized. For any $\omega \in \mathbb{C}_{\neq 0}$ there exists a sequence $\left(x_{n}\right)_{n} \subset \mathcal{V}^{+}$such that $\exp \left(-\frac{1}{2 x_{n}^{2}}\right)=\omega$ and $\left(x_{n}\right)_{n}$ converges towards 0 ; let $y$ be given in order that $\left(x_{n}, y\right)_{n} \subset \Delta$. By assumption $\varphi$ takes the form $\varphi\left(x_{n}, y\right)=\left(X\left(x_{n}\right), Y\left(x_{n}, y\right)\right)$. Since

$$
y_{\alpha, c}^{+}(x)-y_{\alpha, 0}^{+}(x)=c \exp \left(-\frac{1}{2 x^{2}}\right)
$$

does not depend on $\alpha$ we deduce, by setting $c:=y \omega^{-1}$,

$$
Y\left(x_{n}, y\right)=y_{0,0}^{+}\left(X\left(x_{n}\right)\right)+\frac{\psi^{+}\left(y \omega^{-1}\right)}{y}\left(y-y_{\alpha, 0}^{+}\left(x_{n}\right)\right) \exp \left(-\frac{1}{2 X\left(x_{n}\right)^{2}}\right) .
$$

In particular the sequence $\left(\exp \left(-\frac{1}{2} X\left(x_{n}\right)^{-2}\right)\right)_{n}$ converges towards some complex number $\lambda^{+}(\omega) \in \mathbb{C}$. Therefore

$$
\varphi(0, y)=\left(0, \lambda^{+}(\omega) \psi^{+}\left(y \omega^{-1}\right)\right)
$$




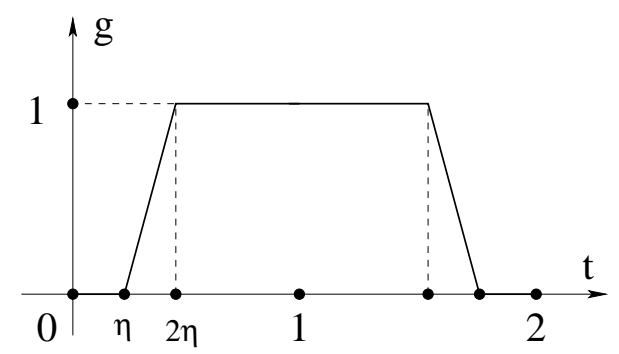

Figure 2.2. The function $g$.

and $\lambda^{+}(\omega) \neq 0$. Obviously the same construction can be carried out on $\mathcal{V}^{-}$, giving rise to a non-zero vanishing function $\omega \mapsto \lambda^{-}(\omega)$ such that for all $y$ one has the same relation $\varphi(0, y)=\left(0, \lambda^{-}(\omega) \psi^{-}\left(y \omega^{-1}\right)\right)$. Hence $\lambda^{+}(\omega) \psi^{+}\left(y \omega^{-1}\right)=$ $\lambda^{-}(\omega) \psi^{-}\left(y \omega^{-1}\right)$ for every $(0, y) \in \Delta$. Now we fix $\omega$ small enough in order that $|\omega|^{-1} \Delta$ contain the points $(0, y) \in \Delta$ and $(0, y+2)$ for at least one value of $y$. Using the 2-periodicity of $\psi^{ \pm}-I d$ we derive

$$
\lambda^{+}(\omega) \psi^{+}(y+2)=\lambda^{-}(\omega) \psi^{-}(y+2)+2\left(\lambda^{+}(\omega)-\lambda^{-}(\omega)\right),
$$

meaning $\lambda^{+}(\omega)=\lambda^{-}(\omega)$. Therefore the first two conditions of $(2.4)$ yields $\psi^{+}(c+1+\alpha)=$ $\psi^{+}(c+1-\alpha)$ for all $c \in \mathbb{C}$. Since $\psi^{+}$is one-to-one the only possibility is $\alpha=0$.

2.4. The transverse homeomorphisms. To go back to our purpose we first find admissible $\psi^{ \pm}$.

Proposition 2.7. Let $|\alpha|<\frac{1}{10}$ and $\eta:=(1-|\operatorname{Re}(\alpha)|) / 3$. We define $g$ as to be the simplest piece-wise real affine map $g$ on $[0,2]$ such that $\left.g\right|_{[0, \eta]}:=0,\left.g\right|_{[2 \eta, 2-2 \eta]}:=1$ and $\left.g\right|_{[2-\eta, 2]}:=0$. We still denote by $g$ its 2 -periodic extension to $\mathbb{R}$. Then the following functions

$$
\begin{aligned}
& c \mapsto \psi^{+}(c):=c+\alpha g(\operatorname{Re}(c)) \\
& c \mapsto \psi^{-}(c):=c-\alpha+\alpha g(1+\operatorname{Re}(c-\alpha))
\end{aligned}
$$

form a pair of homeomorphisms solution to (2.4). Moreover for all $c \in \mathbb{C}$ :

$$
\left|\frac{\psi^{ \pm}(c)}{c}-1\right|<\min \left(\frac{1}{6}, \frac{1}{10|\operatorname{Re}(c)|}\right) .
$$

Proof. The fact that $\left(\psi^{+}, \psi^{-}\right)$is solution to the system $(2.4)$ is clear enough. Besides $|\alpha|<\frac{1}{10}$ so

$$
\sup _{t \in[-1,1]}\left|\frac{g(t)}{t}\right| \leq \frac{1}{2 \eta}<\frac{5}{3},
$$

while for $|t| \geq 1$ one has $g(t) \leq 1$. Hence

$$
\left|\frac{\psi^{+}(c)}{c}-1\right|<|\alpha| \min \left(\frac{5}{3}, \frac{1}{|R e(c)|}\right) \text {. }
$$


The same kind of estimate arises for the other map, where if $|R e(c)| \in[0,1]$ :

$$
\begin{aligned}
\left|\frac{\psi^{-}(c)}{c}-1\right| & =\left|\frac{\alpha}{c}(g(1-\operatorname{Re}(\alpha)+\operatorname{Re}(c))-1)\right| \\
& <\frac{5}{3}|\alpha|<\frac{1}{6}
\end{aligned}
$$

since $1+\operatorname{Re}(\alpha)-\eta>\frac{3}{5}$. To end the proof we only need to notice that $\psi^{ \pm}-I d$ is $\frac{1}{6}$-Lipschitz, thus one-to-one and onto $\mathbb{C}$.

2.5. The homeomorphism $\varphi$. We look now for $\varphi$ on $\overline{\mathbb{D}} \times \mathbb{C}$ as we'll extend it to the entire projective plane in the next section. As was noticed in Proposition 2.6 we cannot preserve globally the $x$-variable in the four directions $\left\{\cos \arg x^{2}=0\right\}$. Hence we build a new (sectorial) variable $X^{ \pm}(x, y)$ which will mostly be the identity except in the neighbourhood of those forbidden directions. We define

$$
X^{ \pm}(x, y):=x\left(1-2 x^{2} \log f^{ \pm}\left(x, c^{ \pm}(x, y)\right)\right)^{-1 / 2}
$$

where $f^{ \pm}$is a functional parameter which will be adjusted in the sequel to suit our needs, and where $c^{ \pm}$is the function holomorphic on $\mathcal{V}^{ \pm}$defined by the relation

$$
y_{\alpha, c^{ \pm}(x, y)}(x)=y \text {. }
$$

For any fixed $x$ the partial function $y \mapsto c^{ \pm}(x, y)$ is a diffeomorphism of $\mathbb{C}$.

In order to send a leave of $\mathcal{F}_{\alpha}^{ \pm}$into a leave of $\mathcal{F}_{0}^{ \pm}$while changing the transverse structure we take the new $y$-variable as being the following

$$
Y^{ \pm}(x, y):=y_{0,0}^{ \pm}\left(X^{ \pm}(x, y)\right)+f^{ \pm}\left(x, c^{ \pm}(x, y)\right) \psi^{ \pm}\left(c^{ \pm}(x, y)\right) \exp \left(-\frac{1}{2 x^{2}}\right)
$$

and set

$$
\varphi^{ \pm}(x, y):=\left(X^{ \pm}(x, y), Y^{ \pm}(x, y)\right)
$$

If we want that $\varphi^{+}$and $\varphi^{-}$glue on each connected component of $\mathcal{V}^{+} \cap \mathcal{V}^{-}$we must require that for all $c \in \mathbb{C}$ :

$$
\left\{\begin{array}{ll}
f^{+}(x, c)=f^{-}(x, c+1+\alpha) & , \forall \operatorname{Re}(x)<0 \\
f^{-}(x, c)=f^{+}(x, c+1-\alpha) & , \forall \operatorname{Re}(x)>0
\end{array} .\right.
$$

If we moreover wish that $\varphi^{ \pm}$extend to $I d$ on $\{x=0\}$ the parameters $f^{ \pm}$must satisfy the additional condition that for all $y_{0}$ :

$$
\lim _{(x, y) \rightarrow\left(0, y_{0}\right)} f^{ \pm}\left(x, c^{ \pm}(x, y)\right) \frac{\psi^{ \pm}\left(c^{ \pm}(x, y)\right)}{c^{ \pm}(x, y)}=1 .
$$

The following lemma is straightforward to prove :

Lemma 2.8. Let $\left(\chi_{1}, \chi_{2}\right)$ be the simplest non-negative affine partition of unity of the circle $\mathbb{S}^{1} \simeq \mathbb{R}_{/ 2 \pi \mathbb{Z}}$ such that $\chi_{1}\left(\frac{\pi}{4}+k \frac{\pi}{2}\right)=1$ for any $k \in \mathbb{Z}_{/ 4 \mathbb{Z}}, \chi_{2}(\theta)=1$ whenever $|\cos (2 \theta)|>\delta$ for some small, fixed $\delta>0$ and $\chi_{1}+\chi_{2}=1$. Define the functions

$$
f^{ \pm}(x, c):=\quad \chi_{1}(\arg x) \frac{c}{\psi^{ \pm}(c)}+\chi_{2}(\arg x) .
$$

These functions satisfy the following properties :

(1) $\left|f^{ \pm}(x, c)-1\right|<\frac{1}{5}$ and $\left|2 x^{2} \log f^{ \pm}(x, c)\right|<\frac{3}{5}|x|^{2}$ whenever $|x| \leq 1$,

(2) $f^{ \pm}$is continuous on $\overline{\mathbb{D}} \times \mathbb{C}$, 
(3) $f^{ \pm}$is constant to 1 on $\mathcal{V}^{+} \cap \mathcal{V}^{-}$,

(4) $\lim _{(x, y) \rightarrow\left(0, y_{0}\right)} f^{ \pm}\left(x, c^{ \pm}(x, y)\right) \frac{\psi^{ \pm}\left(c^{ \pm}(x, y)\right)}{c^{ \pm}(x, y)}=1$ for all $y_{0} \in \mathbb{C}$,

(5) for all fixed $x$ the maps $c \mapsto f^{ \pm}(x, c) \psi^{ \pm}(c)$ are homeomorphisms of the complex line.

As a consequence the map $\varphi$ thus defined is a continuous map from $\overline{\mathbb{D}} \times \mathbb{C}$ conjugating the foliations $\mathcal{F}_{\alpha}$ and $\mathcal{F}_{0}$ on this domain.

Proposition 2.9. The map $\varphi$ is one-to-one and thus defines a homeomorphism from $\overline{\mathbb{D}} \times \mathbb{C}$ onto its image $W \times \mathbb{C}$ which, up to rescaling $\varphi$ in the first coordinate for both source and target spaces, contains $\overline{\mathbb{D}} \times \mathbb{C}$.

Proof. Firstly we shall prove the latter claim. Let us write $\varphi=(X, Y)$. Because of the first statement of the previous lemma we have

$$
\left|\frac{X(x, y)}{x}-1\right| \leq A|x|^{2}
$$

for some $A>0$ and all $|x| \leq 1$. This implies that for $|x|<\delta$ small enough $\varphi\left(\mathcal{V}^{ \pm} \cap \delta \mathbb{D} \times \mathbb{C}\right)$ contains a sector $W^{ \pm}:=\left\{x:|x|<r,\left|\arg x \mp \frac{\pi}{2}\right|<\frac{3 \pi}{4}-\theta\right\}$, where $\theta$ can be chosen as small as we wish by decreasing $\delta$. Up to rescalling the $x-$ and $X$-coordinates we can then assume that $\overline{\mathbb{D}} \subset X(\overline{\mathbb{D}} \times \mathbb{C})$. Because of $(5)$ we also derive that $\varphi\left(\mathcal{V}^{ \pm} \cap \delta \mathbb{D} \times \mathbb{C}\right)$ contains a sector of lesser aperture $W^{ \pm} \times \mathbb{C}$, so that $\overline{\mathbb{D}} \times \mathbb{C} \subset \varphi(\overline{\mathbb{D}} \times \mathbb{C})$ as required.

To prove that $\varphi$ is one-to-one we first notice that since $\varphi$ preserves the sector decomposition of $\overline{\mathbb{D}} \times \mathbb{C}$ and since each leaf of the sectorial foliations $\mathcal{F}_{\alpha}^{ \pm}$is the graph of a function holomorphic on $W^{ \pm}$, we only need to prove that the restriction of $\varphi^{ \pm}$to some transversal $\left\{x=x_{0}\right\}$ is one-to-one. Let us choose $x_{0}:= \pm 1$, so that $f^{ \pm}\left(x_{0}, c\right)=1$. We thus have that

$$
\varphi^{ \pm}\left(x_{0}, y\right)=\left(x_{0}, y_{0,0}\left(x_{0}\right)+\psi^{ \pm}\left(c^{ \pm}\left(x_{0}, y\right)\right) \exp \left(-\frac{1}{2 x_{0}^{2}}\right)\right)
$$

which completes the proof.

\section{EXTENDING THE HOMEOMORPHISM}

The foliations under consideration have exactly three singularities, located in homogeneous coordinates at $[0: 0: 1],[0: 1: 0]$ and $[1: 0: 0]$. We will use the following three affine charts of $\mathbb{C} P^{2}$ :

$$
\begin{aligned}
& \mathbb{C}^{2}=\{(x, y)\}=\{[x: y: 1]\} \\
& \mathbb{C}^{2}=\{(s, t)\}=\{[1: t: s]\} \\
& \mathbb{C}^{2}=\{(u, v)\}=\{[u: 1: v]\}
\end{aligned}
$$

with transition maps

$$
y=t x, 1=s x, x=u y, 1=v y, v=u s, 1=u t \quad .
$$

The singular point $[0: 0: 1]$ has been extensively studied in the previous sections and this study gave rise to a topological conjugacy between the foliations $\mathcal{F}_{0}$ and $\mathcal{F}_{\alpha}$ from $\overline{\mathbb{D}} \times \mathbb{C}$ onto its image.

Lemma 3.1. One can extend $\varphi$ to a homeomorphism of $\mathbb{C} \times \mathbb{C}$ such that the extension, still noted $\varphi$, preserves each fiber of $\Pi$ outside $\overline{\mathbb{D}} \times \mathbb{C}$ and still conjugates $\mathcal{F}_{0}$ and $\mathcal{F}_{\alpha}$. 
Proof. This result is straightforward. Choose $0<r<1$ and consider the simplest affine non-negative partition of unity $\left(\xi_{1}, \xi_{2}\right)$ of $\mathbb{R}_{\geq 0}$ where $\xi_{1}=1$ on $[0, r], \xi_{2}=1$ outside $[0,1]$ and $\xi_{1}+\xi_{2}=1$. By setting

$$
\begin{array}{ll}
(\forall(x, c) \in \overline{\mathbb{D}} \times \mathbb{C}) & \hat{f}^{ \pm}(x, c):=f^{ \pm}(x, c) \xi_{1}(|x|)+\xi_{2}(|x|) \\
(\forall(x, c) \notin \overline{\mathbb{D}} \times \mathbb{C}) & \hat{f}^{ \pm}(x, c):=1
\end{array}
$$

and defining $\varphi$ by (2.7) the reader can easily show that our claim is true, as in Proposition 2.8.

Once this is stated we have to check that $\varphi$ extends to a homeomorphism of $\mathbb{C} P^{2}$. It is only a matter of writing things in appropriate charts since as expected $\varphi$ extends to $I d$ along the line at infinity $\{s=0\} \cup\{v=0\}$.

Proposition 3.2. $\varphi$ extends to a global homeomorphism of $\mathbb{C} P^{2}$, which implies that the main theorem is true.

Proof. Let us write $\varphi$ in the chart $(s, t)$ near $\{0\} \times \mathbb{C}$ :

$$
\tilde{\varphi}(s, t):=\left(s, s\left(y_{0,0}^{ \pm}\left(\frac{1}{s}\right)+\psi^{ \pm}\left(\tilde{c}^{ \pm}(s, t)\right) \exp \left(-\frac{s^{2}}{2}\right)\right)\right)
$$

where as before $\tilde{c}^{ \pm}$is uniquely defined on $\mathcal{V}^{ \pm}$by

$$
y_{\alpha, \tilde{c}^{ \pm}(s, t)}^{ \pm}\left(\frac{1}{s}\right)=\frac{t}{s},
$$

for all $(s, t) \in \mathbb{C}_{\neq 0} \times \mathbb{C}$. Following (2.3) we have

$$
y_{\alpha, 0}^{ \pm}\left(\frac{1}{s}\right)=-\exp \left(-\frac{s^{2}}{2}\right) \int_{s}^{ \pm \infty i}\left(1+\frac{\alpha}{z}\right) \exp \left(\frac{z^{2}}{2}\right) \frac{\mathrm{d} z}{z}
$$

so that

$$
\lim _{s \rightarrow 0} s y_{\alpha, 0}^{ \pm}\left(\frac{1}{s}\right)=0 .
$$

On the other hand if $(s, t) \in \mathcal{V}^{ \pm}$then $t=s\left(y_{0,0}^{ \pm}\left(\frac{1}{s}\right)+\tilde{c}^{ \pm}(s, t) \exp \left(-s^{2} / 2\right)\right)$ so that setting

$$
\tilde{\varphi}(0, t):=(0, t)
$$

defines a continuous extension of $\tilde{\varphi}$ to $\mathbb{C} \times \mathbb{C}$, because $\psi^{ \pm}\left(\tilde{c}^{ \pm}(s, t)\right)-\tilde{c}^{ \pm}(s, t)$ remains bounded as $(s, t) \rightarrow\left(0, t_{0}\right)$.

Finally we shall check that $\varphi$ admits a limit at $[0: 1: 0]$. We write it in the chart $(u, v)$ as

$$
\hat{\varphi}(u, v):=\left(\hat{U}^{ \pm}(u, v), \hat{V}^{ \pm}(u, v)\right)
$$

where

$$
\begin{aligned}
\hat{X}^{ \pm}(u, v) & =\frac{u}{v \sqrt{1-2 \frac{u^{2}}{v^{2}} \log f^{ \pm}\left(\frac{u}{v}, \hat{c}^{ \pm}(u, v)\right)}} \\
\left(3 . \hat{\mathbb{Y}}^{ \pm}(u, v)\right. & =\frac{1}{y_{0,0}^{ \pm}\left(\hat{X}^{ \pm}(u, v)\right)+f^{ \pm}\left(\frac{u}{v}, \hat{c}^{ \pm}(u, v)\right) \psi^{ \pm}\left(\hat{c}^{ \pm}(u, v)\right) \exp \left(-\frac{v^{2}}{2 u^{2}}\right)} \\
\hat{U}^{ \pm}(u, v) & =\hat{V}^{ \pm}(u, v) \hat{X}^{ \pm}(u, v)
\end{aligned}
$$


and $\hat{c}^{ \pm}(u, v)$ is uniquely defined by $v^{-1}=y_{\alpha, 0}(u / v)+\hat{c}^{ \pm}(u, v) \exp \left(-v^{2} / 2 u^{2}\right)$ for $\left(\frac{u}{v}, \frac{1}{v}\right) \in \mathcal{V}^{ \pm}$. Let us split $\mathbb{C}_{\neq 0}^{2}$ into the sets

$$
\begin{aligned}
\mathcal{C}^{>}: & =\left\{(u, v):\left|\frac{u}{v}\right|>1\right\} \\
\mathcal{C} \leq & :=\left\{(u, v):\left|\frac{u}{v}\right| \leq 1\right\} .
\end{aligned}
$$

We recall that $f^{ \pm}\left(\frac{u}{v}, \hat{c}^{ \pm}(u, v)\right)=1$ whenever $(u, v) \in \mathcal{C}^{>}$. On the other hand $\hat{X}^{ \pm}(\mathcal{C} \leq)$ is bounded. Hence it suffices to show that $\hat{V}^{ \pm}(u, v)=O(v)$ as $(u, v) \rightarrow 0$ in order to prove that $\hat{\varphi}$ extends continuously to $(0,0)$ by $\hat{\varphi}(0,0):=(0,0)$.

Because $x \mapsto y_{0,0}^{ \pm}(x)$ is smooth as a real map there exists a constant $A>0$ such that for all $(u, v) \in \mathcal{C}^{<}$one has

$$
\left|y_{0,0}^{ \pm}\left(\hat{X}^{ \pm}(u, v)\right)-y_{0,0}^{ \pm}\left(\frac{u}{v}\right)\right| \leq A\left|\frac{u^{3}}{v^{3}}\right|
$$

whereas this estimate is true with $A:=0$ when $(u, v) \in \mathcal{C}^{>}$. We then derive :

$$
\begin{aligned}
& \left|\frac{1}{\hat{V}^{ \pm}(u, v)}-\frac{1}{v}\right| \leq A\left|\frac{u^{3}}{v^{3}}\right|+\left|\hat{c}^{ \pm}(u, v)-f^{ \pm}\left(\frac{u}{v}, \hat{c}^{ \pm}(u, v)\right) \psi^{ \pm}\left(\hat{c}^{ \pm}(u, v)\right)\right|\left|\exp \left(-v^{2} / 2 u^{2}\right)\right| \\
& \left|\frac{v}{\hat{V}^{ \pm}(u, v)}-1\right| \leq A|u|\left|\frac{u}{v}\right|^{2}+B|v|\left|\exp \left(-v^{2} / 2 u^{2}\right)\right|
\end{aligned}
$$

for some $B>0$. Hence there exists $B^{>}>0$ such that for all $(u, v) \in \mathcal{C}^{>}$:

$$
\left|\frac{v}{\hat{V}^{ \pm}(u, v)}-1\right|<B^{>}|v| .
$$

Consider now $(u, v) \in \mathcal{C} \leq$. Clearly there exists $B_{+}^{\leq}>0$ such that if $\operatorname{Re}\left(\frac{u^{2}}{v^{2}}\right) \geq 0$ then

$$
\left|\frac{v}{\hat{V}^{ \pm}(u, v)}-1\right|<B_{+}^{\leq}(|u|+|v|)
$$

while according to (3.1) there exists $B_{-}^{\leq}>0$ such that if $\operatorname{Re}\left(\frac{u^{2}}{v^{2}}\right)<0$ then

$$
\left|\hat{V}^{ \pm}(u, v)\right|<B_{-}^{\leq}|v|
$$

\section{REFERENCES}

[E] P. M. Elizarov, Orbital topological classification of analytic differential equations in a neighbourhood of a degenerate elementary singular point on the two-dimensional complex plane, Trudy Sem. Petrovskogo, vol.13, p. 137-165 (1988)

[GO] X. Gómez-Mont, L. Ortíz-Bobadilla, Sistemas dinámicos holomorfos en superficies, Sociedad Matemática Mexicana, México City (1989)

[I] Yu. S. Ilyashenko, Topology of phase portraits of analytic differential equations on a complex projective plane, Trudy Sem. Petrovskogo, vol.4, p. 83-136 (1978)

[L] N. N. Ladis, Integral curves of complex homogenuous equations, Differential Equations, vol.15 \#2, p. 167-171 (1979)

[N] I. Nakai, Separatrices for nonsolvable dynamics on $(\mathbb{C}, 0)$, Annales de l'Institut Fourier, vol.44 \#2, p. $569-599$ (1994) 
[NSS] A. L. Neto, P. Sad, B. Scárdua, On topological rigidity of projective foliations, Bulletin de la Société Mathématique de France, vol.126 \#3, p. 381-406 (1998)

[M] J.-F. Mattéi, Modules de feuilletages holomorphes singuliers. I. Équisingularité., Inventiones Mathematicae, vol.103 \#2, p. 297-325 (1991)

[MR] J. Martinet, J.-P. Ramis, Problèmes de modules pour des équations différentielles non linéaires du premier ordre. Institut des Hautes Études Scientifiques, Publications Mathématiques, vol. 55, p. 63-164 (1982)

[S] A. A. Scherbakov, Topological and analytical conjugation of noncommutative groups of germs of conformal mappings, Journal Soviet Mathematics, vol.35 \#6, p. 2827-2850 (1986)

[T] L. Teyssier, Examples of non-conjugated holomorphic vector fields and foliations, Journal of Differential Equations, vol.205 \#2, p. 390-407 (2004)

[V] S. M. Voronin, Smooth and analytic equivalence of germs of resonance vector fields and maps, Usp. Mat. Nauk, vol.38 \#5, p. 111-126 (1983)

Institut de Recherche Mathématique Avancée (UMR 7501), Université de Strasbourg et C.N.R.S., 7 rue René Descartes, 67000 Strasbourg, France 